\title{
Proteção radiológica: A reflexão da ergologia sobre o trabalho em saúde
}

\author{
Radiological protection: The reflection of ergology on health work \\ Protección radiológica: El reflejo de la ergología en el trabajo sanitario
}

Recebido: 06/06/2021 | Revisado: 15/06/2021 | Aceito: 17/06/2021 | Publicado: 02/07/2021

Ezequiel da Silva

ORCID: https://orcid.org/0000-0002-0233-435X Instituto Federal de Educação, Ciência e Tecnologia de Santa Catarina, Brasil E-mail: zequi.silva@gmail.com

Gerusa Ribeiro

ORCID: https://orcid.org/0000-0003-3188-8017 Instituto Federal de Educação, Ciência e Tecnologia de Santa Catarina, Brasil E-mail: gerusa@ifsc.edu.br

Juliana Almeida Coelho de Melo

ORCID: https://orcid.org/0000-0003-2217-9649 Instituto Federal de Educação, Ciência e Tecnologia de Santa Catarina, Brasil E-mail: julianac@ifsc.edu.br

Rosane Seeger da Silva

ORCID: https://orcid.org/0000-0001-7980-4762 Universidade Federal de Santa Maria, Brasil E-mail: rosane.seeger@hotmail.com

Suzinara Beatriz Soares de Lima

ORCID: https://orcid.org/0000-0002-2162-8601 Universidade Federal de Santa Maria, Brasil E-mail: suzibslima@yahoo.com.br

Caroline de Medeiros

ORCID: https://orcid.org/0000-0002-1469-8724 Instituto Federal de Educação, Ciência e Tecnologia de Santa Catarina, Brasil E-mail: carol@ifsc.edu.br

\section{Resumo}

A Ergologia é a perspectiva científica e filosófica que estuda o trabalho humano buscando contemplar a atividade humana em todas as suas dimensões, baseada no projeto de melhor conhecer o trabalho para intervir e transformá-lo. Trata-se de um estudo de revisão integrativa de literatura cujo objetivo foi discorrer e refletir acerca da Ergologia como referencial teórico e suas contribuições no trabalho em saúde e na proteção radiológica. A Ergologia como referencial teórico é de extrema importância e aplicação prática. Há que se intensificar a execução de suas práticas nas pesquisas e na aplicabilidade na Proteção Radiológica, ampliando e maximizando a qualidade da assistência prestada aos pacientes bem como a qualidade de vida dos trabalhadores e usuários nos serviços de saúde.

Palavras-chave: Ergologia; Proteção radiológica; Trabalho; Profissionais de saúde.

\begin{abstract}
Ergology is the scientific and philosophical perspective that studies human work seeking to contemplate human activity in all its dimensions, based on the project of better knowing the work to intervene and transform it. This is an integrative literature review study whose objective was to discuss and reflect on Ergology as a theoretical framework and its contributions to health work and radiological protection. Ergology as theoretical framework is of extreme importance and practical application. It is necessary to intensify the execution of its practices in research and in the applicability in Radiological Protection, expanding and maximizing the quality of care provided to patients as well as the quality of life of workers and users in health services.
\end{abstract}

Keywords: Ergology; Radiological protection; Work; Health professionals.

\section{Resumen}

La ergología es la perspectiva científica y filosófica que estudia el trabajo humano buscando contemplar la actividad humana en todas sus dimensiones, partiendo del proyecto de conocer mejor el trabajo para intervenir y transformarlo. Se trata de un estudio de revisión integradora de la literatura cuyo objetivo fue discutir y reflexionar sobre la Ergología como marco teórico y sus aportes al trabajo de salud y protección radiológica. La ergología como marco teórico es de extrema importancia y aplicación práctica. Es necesario intensificar la ejecución de sus prácticas en investigación y en la aplicabilidad en Protección Radiológica, ampliando y maximizando la calidad de la atención brindada a los pacientes así como la calidad de vida de los trabajadores y usuarios de los servicios de salud.

Palabras clave: Ergología; Protección Radiológica; Trabajo, Profesionales de la salud. 


\section{Introdução}

A abordagem ergológica surgiu na França, na década de 1980, impulsionada pelo cenário de mudanças socioeconômicas no mundo do trabalho e na sociedade europeia (Schwartz, 2006). É uma perspectiva científica e filosófica que estuda o trabalho humano buscando contemplar a atividade humana em todas as suas dimensões, baseada no projeto de melhor conhecer o trabalho para intervir e transformá-lo. Ela parte da análise cuidadosa dos estudos indicativos da distância entre o trabalho prescrito e o real; nela o trabalhador procura agir nas situações de trabalho para que haja contribuição para permitir ao trabalhador crescer, pois possui como pontos de partida e chegada a atividade humana (Lima \& Bianco, 2009; Athayde \& Brito, 2011; Hennington; Cunha \& Fischer, 2011).

O referencial da ergologia tem sido útil nos estudos que envolvem o processo de trabalho em saúde, seja como método ou teoria, com o intuito de melhor compreender e estimular a complexidade da atividade desenvolvida pelos profissionais de saúde. Os trabalhadores de saúde de um modo geral, encontram-se inseridos em ambientes que representam risco à sua saúde, requer tomada de decisão imediata, influenciadas e ditadas por normas, protocolos e as políticas públicas prescritas. Em constante movimento, em um meio infiel, não dissociada do seu agir, requer recriar normas e a renormalização, com a competência técnica e legal do cuidado à saúde da população (Ribeiro \& Pires et. al, 2019).

Dentre os processos de trabalho dos profissionais de saúde está o "serviço de diagnóstico por imagem, um dos mais importantes instrumentos de apoio a inúmeras áreas da medicina" (Fernandes et. al, 2005), onde são observados atos inseguros e condições ambientais de insegurança, como por exemplo: preparação e manuseio de soluções tóxicas sem utilização de equipamento de proteção individual (EPI); trabalhadores e usuários em ambientes onde há periculosidade, resultante da detecção de níveis de radiações ionizantes acima dos limites estabelecidos por lei.

A radiação pode ser dividida em: radiação ionizante e radiação não-ionizante. Conforme define CNEN (2006), a radiação ionizante é qualquer partícula ou radiação eletromagnética que ao interagir com a matéria, ioniza seus átomos ou moléculas. Os profissionais dos serviços de saúde, mais precisamente os que trabalham em setores de diagnóstico por imagens e, setores que utilizam a radiação ionizante como tratamento, nos ambientes hospitalares, estão mais expostos às radiações ionizantes e, faz-se necessário implementar um conjunto de medidas que busquem protegê-los. Além disso, tais medidas devem também garantir a proteção aos usuários e seus acompanhantes evitando os efeitos probabilísticos da radiação. Esse conjunto de medidas é denominado Proteção Radiológica ou Radioproteção.

O conceito amplo de radioproteção considera a prevenção dos possíveis danos provocados pelas radiações ionizantes, que dependem da dose de radiação absorvida, em consequência da carga de cada exposição e do acúmulo de cargas repetidas. Assim, é considerado como radioproteção todo cuidado que reduz a exposição à radiação ionizante, tais como a indicação de exames radiológicos adequados e indispensáveis, a utilização da mínima dose necessária de radiação para realizar o exame com qualidade e restringindo a exposição as áreas de interesse e o uso de equipamentos de proteção individual para o técnico e para as pessoas que estão nas proximidades e, por algum motivo, não podem se afastar da fonte de radiação (Moores, 2017; Kase, 2004).

De acordo com Pereira (2004), o objetivo primário da Proteção Radiológica é proporcionar um padrão de proteção para o homem, sem limitar as práticas benéficas que envolvem exposição à radiação. A Comissão Internacional de Proteção Radiológica (ICRP, 1990) recomenda que providências devam ser tomadas para reduzir exposições desnecessárias.

Assim, em concordância com o dinamismo do trabalho de diagnóstico por imagem, a Ergologia desponta como um referencial teórico-metodológico na perspectiva de contemplar, dentre outros, o forte aspecto relacional da interação entre os trabalhadores e o ambiente de trabalho, inerentes à profissão.

$\mathrm{O}$ presente estudo foi motivado pela necessidade de divulgação e ampliação das discussões acerca do referencial da ergologia, como teoria e método, que subsidiam a pesquisa envolvendo profissionais de saúde. Desta forma desenvolveu-se o 
seguinte questionamento: Quais as contribuições teóricas ou metodológicas, o referencial da ergologia tem sido útil na compreensão da atividade humana dos profissionais de saúde, em especial na área de proteção radiológica?

\section{Metodologia}

Este estudo é um recorte da Dissertação de Mestrado Profissional em Proteção Radiológica, intitulada "Proteção Radiológica em uma Unidade Cirúrgica: o prescrito e o realizado". Trata-se de um estudo de revisão integrativa, de natureza qualitativa (Pereira et al. 2018), na qual a reflexão foi realizada com base na literatura nacional acerca da temática, constituída de artigos científicos. Esse método busca reunir e sintetizar resultados de estudos sobre um delimitado tema ou questão (Pompeo; Rossi \& Galvão, 2009).

Os métodos para a condução de revisões integrativas apresentam variações, contudo, com alguns padrões a serem seguidos (Silveira \& Zago, 2006). Neste estudo, utilizaram-se seis etapas: a) identificação do tema e a questão de pesquisa para a elaboração da revisão integrativa; b) estabelecimento de critérios para inclusão e exclusão de estudos e busca na literatura; c) definição das informações a serem extraídas dos estudos selecionados e a categorização dos estudos; d) avaliação dos estudos incluídos na revisão integrativa; e) interpretação dos resultados e apresentação da revisão; f) síntese do conhecimento (Mendes; Silveira \& Galvão, 2008).

A busca foi realizada na Biblioteca Virtual da Saúde (BVS), com acesso a rede privada virtual, VPN, (Virtual Private Network) do Instituto Federal de Educação, Ciência e Tecnologia de Santa Catarina, entre os meses de outubro e novembro de 2020. Foram usadas as seguintes bases de dados: Scientific Electronic Library Online (SCIELO), da Base de Dados de Enfermagem (BDENF) e da Literatura Latino-Americana e do Caribe em Ciências da Saúde (LILACS) e as palavras-chaves e/ou descritores: "Ergologia", "Proteção Radiológica", "Trabalho" e "Pessoal de saúde" associadas ao uso do conector boleano AND. Sendo utilizados somente termos em português, a ampliação de palavras-chaves em inglês, limitou a obtenção das publicações.

Para a inclusão dos artigos, foram empregados os seguintes critérios: texto na íntegra, delimitação temporal (2000 a 2020), população-alvo (profissionais de saúde), tipo de estudo (sem delimitação) e idioma português. Tais estratégias foram tomadas com o intuito de maximizar os resultados da pesquisa, uma vez que foi constatada escassez de literatura, no contexto nacional, já que no contexto internacional, não foram encontrados resultados. Foram excluídos os estudos que não obedeceram aos critérios de inclusão supracitados, os que não estavam liberados de forma gratuita, e os artigos repetidos. Optou-se por utilizar como material apenas artigos científicos devido à facilidade de acesso a este tipo de publicação.

As associações dos descritores e das palavras-chaves, resultaram em 29 indicações, das quais apenas 10 artigos atendiam na íntegra o interesse da pesquisa, registrando-se, ainda, que alguns não contemplavam a temática abordada.

Posteriormente ao processo de seleção dos artigos, todos os títulos e após, todos os resumos foram lidos, para avaliação e exclusão dos que não correspondiam aos objetivos do estudo, dito de primeira peneira. Nos casos em que a leitura do resumo não era suficiente para estabelecer se o artigo deveria ser incluído, considerando-se os critérios de inclusão definidos, o artigo foi lido na íntegra para determinar sua elegibilidade, definido por segunda peneira. Quando o resumo era suficiente, os artigos eram selecionados e, então, obtida a versão integral para confirmação de elegibilidade e inclusão no estudo.

As informações relevantes das publicações selecionadas foram extraídas e sumarizadas em quadros denominados: "Identificação e descrição de estudos que discorrem sobre ergologia e o trabalho em saúde" e "Identificação e descrição de estudos que discorrem sobre proteção radiológica e o trabalho em saúde".

Depois de realizada essa etapa, iniciou-se a redação do presente estudo de revisão, tornando possível articular as diferentes representações que propiciaram o entendimento sobre o assunto. 
Por tratar-se de uma pesquisa bibliográfica, com uso exclusivo de bases de dados públicos, esta dispensa a submissão ao Comitê de Ética em Pesquisa em Seres Humanos. Abaixo apresentam-se os respectivos resultados.

\section{Resultados e Discussão}

As publicações foram classificadas por título, objetivo, autores, revista, ano e a base de dados, conforme expõe o Quadro 1.

Quadro 1 - Identificação e descrição de estudos que discorrem sobre ergologia e o trabalho em saúde 2008-2019.

\begin{tabular}{|c|c|c|c|c|}
\hline Título & Autores & $\begin{array}{c}\text { Periódico/ano de } \\
\text { publicação }\end{array}$ & $\begin{array}{c}\text { Base de } \\
\text { dados }\end{array}$ & Objetivo do estudo \\
\hline $\begin{array}{l}\text { Gestão dos processos de } \\
\text { trabalho e humanização } \\
\text { em saúde: reflexões a } \\
\text { partir da ergologia }\end{array}$ & Hennington, É. A. & $\begin{array}{l}\text { Rev. Saúde } \\
\text { Pública/2008 }\end{array}$ & MEDLINE & $\begin{array}{l}\text { Refletir sobre o trabalho em } \\
\text { saúde, buscando confrontar o } \\
\text { pensamento do filósofo francês } \\
\text { Schwartz e a temática da gestão } \\
\text { dos processos de trabalho no } \\
\text { contexto da política de } \\
\text { humanização. }\end{array}$ \\
\hline $\begin{array}{l}\text { A incorporação da } \\
\text { abordagem ergológica } \\
\text { na formação dos } \\
\text { profissionais de saúde: } \\
\text { em busca da } \\
\text { integralidade da atenção } \\
\text { à saúde }\end{array}$ & Neves, T. P. & Rev. APS/2010 & LILACS & $\begin{array}{l}\text { Discutir como os referenciais da } \\
\text { ergologia podem contribuir para } \\
\text { que a formação dos } \\
\text { profissionais de saúde seja } \\
\text { direcionada à obtenção da } \\
\text { integralidade da atenção à saúde }\end{array}$ \\
\hline $\begin{array}{l}\text { Um olhar sobre o } \\
\text { trabalho de enfermagem } \\
\text { e a ergologia }\end{array}$ & $\begin{array}{l}\text { Santos, T. M. \& } \\
\text { Camponogara, S. }\end{array}$ & $\begin{array}{l}\text { Trab. Educ. } \\
\text { Saúde/2014 }\end{array}$ & LILACS & $\begin{array}{l}\text { Identificar e analisar artigos } \\
\text { científicos, teses e dissertações } \\
\text { que tratem de subjetividade no } \\
\text { trabalho em enfermagem, com } \\
\text { destaque especial para as } \\
\text { investigações fundamentadas no } \\
\text { referencial teórico- } \\
\text { metodológico da ergologia no } \\
\text { Brasil. }\end{array}$ \\
\hline $\begin{array}{l}\text { Contribuições teórico- } \\
\text { metodológicas da } \\
\text { Ergologia para a } \\
\text { pesquisa sobre o } \\
\text { trabalho da enfermagem }\end{array}$ & $\begin{array}{l}\text { Ribeiro, G.; } \\
\text { Pires, D. E. P. \& } \\
\text { Scherer, M. D. A. }\end{array}$ & $\begin{array}{l}\text { Texto \& Contexto } \\
\text { Enfermagem } / 2019\end{array}$ & SCIELO & 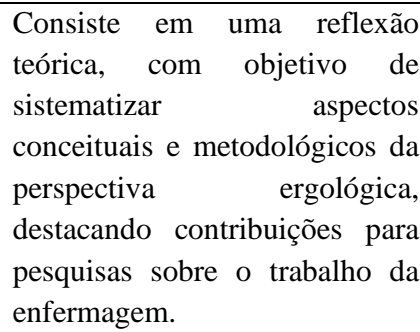 \\
\hline
\end{tabular}

Fonte: Autores (2021).

A ergologia tem como perspectiva a pluridisciplinaridade, com isso buscou-se publicações que articularam a abordagem com o trabalho em saúde a proteção radiológica. O Quadro 2 expõe essa relação. 
Quadro 2 - Classificação dos artigos que articulam o referencial sobre proteção radiológica e o trabalho em saúde de 2011 2019.

\begin{tabular}{|c|c|c|c|c|}
\hline Título & Autores & $\begin{array}{l}\text { Periódico/ano } \\
\text { de publicação }\end{array}$ & $\begin{array}{l}\text { Base de } \\
\text { dados }\end{array}$ & Objetivo do estudo \\
\hline $\begin{array}{l}\text { A saúde } \quad \text { do } \\
\text { trabalhador } \quad \text { em } \\
\text { radiologia: algumas } \\
\text { considerações }\end{array}$ & $\begin{array}{l}\text { Brand, C. I.; } \\
\text { Fontana, R. T. \& } \\
\text { Santos, A. V. }\end{array}$ & $\begin{array}{l}\text { Texto \& } \\
\text { Contexto Enferm. } \\
12011\end{array}$ & $\begin{array}{l}\text { LILACS } \\
\text { BDENF } \\
\text { Enferm. }\end{array}$ & $\begin{array}{l}\text { Identificar sinais e sintomas } \\
\text { autorreferidos por profissionais que } \\
\text { trabalham em serviços de radiologia e os } \\
\text { conhecimentos acerca da radioproteção e } \\
\text { legislação. }\end{array}$ \\
\hline $\begin{array}{l}\text { Melhoria da proteção } \\
\text { radiológica mediante } \\
\text { um ciclo de avaliação } \\
\text { interna da qualidade }\end{array}$ & $\begin{array}{l}\text { Figueiredo, F. M. } \\
\text { \& Gama, Z. A. S. }\end{array}$ & $\begin{array}{l}\text { Radiol Bras } \\
\text { [online]/2012 }\end{array}$ & $\begin{array}{l}\text { SCIELO } \\
\text { LILACS }\end{array}$ & $\begin{array}{l}\text { Determinar o nível de qualidade da } \\
\text { proteção radiológica para os pacientes } \\
\text { durante a realização de exames } \\
\text { radiológicos e avaliar a efetividade de } \\
\text { uma intervenção dirigida a melhorar a } \\
\text { qualidade. }\end{array}$ \\
\hline \begin{tabular}{lr}
\multicolumn{2}{l}{ Proteção radiológica e } \\
a atitude & de \\
trabalhadores & de \\
enfermagem & em \\
serviço & de \\
hemodinâmica. &
\end{tabular} & $\begin{array}{l}\text { Flôr, R. de C. \& } \\
\text { Gelbcke, F. L. }\end{array}$ & $\begin{array}{l}\text { Texto \& } \\
\text { Contexto Enferm. } \\
12013\end{array}$ & $\begin{array}{l}\text { LILACS } \\
\text { BDENF } \\
\text { Enferm. }\end{array}$ & $\begin{array}{l}\text { Analisar a atitude dos trabalhadores de } \\
\text { enfermagem em relação ao uso das } \\
\text { medidas de radioproteção } \\
\text { procedimentos intervencionistas }\end{array}$ \\
\hline $\begin{array}{l}\text { Exposição da equipe } \\
\text { de Enfermagem à } \\
\text { radiação em centro } \\
\text { cirúrgico: um estudo } \\
\text { descritivo }\end{array}$ & $\begin{array}{l}\text { Querido, F. M. \& } \\
\text { Poveda, V. B. }\end{array}$ & $\begin{array}{l}\text { Rev. } \\
\text { SOBECC/2015 }\end{array}$ & LILACS & $\begin{array}{l}\text { Verificar a exposição à radiação da equipe } \\
\text { de Enfermagem nos Centros Cirúrgicos } \\
\text { de sete hospitais de seis cidades do } \\
\text { interior do Estado de São Paulo. }\end{array}$ \\
\hline $\begin{array}{l}\text { Investigação da práxis } \\
\text { em enfermagem } \\
\text { radiológica: aplicação } \\
\text { da metodologia da } \\
\text { psicodinâmica do } \\
\text { trabalho }\end{array}$ & $\begin{array}{l}\text { Flôr, R. de C.; } \\
\text { Melo, J. A. C.; } \\
\text { Gelbcke, F. L.; } \\
\text { Ramos, F. R. S. \& } \\
\text { Amadiji, F. R. }\end{array}$ & $\begin{array}{l}\text { Texto contexto - } \\
\text { enferm. } / 2017\end{array}$ & $\begin{array}{l}\text { SCIELO } \\
\text { LILACS } \\
\text { BDENF }\end{array}$ & $\begin{array}{l}\text { Relatar a utilização do Método da } \\
\text { Psicodinâmica do Trabalho, destacando } \\
\text { os caminhos percorridos desde a demanda } \\
\text { até a interpretação dos dados, de duas } \\
\text { pesquisas distintas realizadas com } \\
\text { trabalhadores de enfermagem em } \\
\text { hemodinâmica e medicina nuclear. }\end{array}$ \\
\hline $\begin{array}{l}\text { A proteção radiológica } \\
\text { na perspectiva dos } \\
\text { profissionais de saúde } \\
\text { expostos à radiação }\end{array}$ & $\begin{array}{l}\text { Batista, V. M. D.; } \\
\text { Bernardo, M. O.; } \\
\text { Morgado, F. \& } \\
\text { Almeida, F. A. }\end{array}$ & $\begin{array}{c}\text { Rev. Bras. } \\
\text { Enferm/2019 }\end{array}$ & SCIELO & $\begin{array}{l}\text { Avaliar o conhecimento dos profissionais } \\
\text { de saúde sobre proteção radiológica e } \\
\text { implementar ações educativas que } \\
\text { promovam um ambiente de trabalho } \\
\text { seguro para profissionais, pacientes e } \\
\text { acompanhantes. }\end{array}$ \\
\hline
\end{tabular}

Fonte: Autores (2021).

\section{Discussão}

Os resultados foram construídos, no conjunto das publicações, sendo categorizados como: Reflexões do trabalho em saúde na abordagem da ergologia; olhar da proteção radiológica sobre o trabalho em saúde; A proteção radiológica interpretada à luz da ergologia.

\subsection{Reflexão do trabalho em saúde na abordagem da ergologia}

A Ergologia - assim chamada a partir dos anos 1990 e apresentada como "[...] um projeto de melhor conhecer e, sobretudo, de melhor intervir sobre as situações de trabalho para transformá-las" (Schwartz, 2010, p. 37) - tem suas origens 
atreladas a estudos pluridisciplinares a respeito de situações de trabalho em fins de 1970 e início de 1980 na França, e é fruto de experimentações e debates entre profissionais do conceito e protagonistas da atividade de trabalho (Athayde \& Brito, 2011; Hennington, Cunha \& Fischer, 2011).

Segundo, Durrive e Jacques (2010, p. 295) "ergo" origina-se de uma palavra grega que significa "ação, trabalho, obra", sinalizando para a energia e vitalidade do trabalhador. Assim, na Ergologia, portanto, a análise é feita tanto quanto possível "do ponto de vista daquele que trabalha" e se constitui no intuito de investigar o permanente debate de normas e de valores que renovam indefinidamente a atividade humana, concentrando-se sobre a relação que a pessoa estabelece com o meio no qual está engajada (Schwartz; Duc \& Durrive, 2010), tendo como objetivo "[...] abrir ao máximo o ângulo sobre todas as dimensões da atividade (matriz do viver), ao mesmo tempo analisando-a à lupa" (Athayde \& Brito, 2011, p. 258).

Para os trabalhadores, o trabalho pode ser visto como o conjunto de atos e operações da pessoa que a executa, implicando na disposição da energia humana que envolve as dimensões físicas, psíquicas, emocionais e sociais. Por isso, trabalhar não é só uma operacionalização no sentido técnico ou mecanizado, mas constitui o ser humano (Ribeiro \& Pires et. al, 2019; Silva, 2009, p.71).Para discorrer sobre o trabalhador e as questões por ele engendradas, a Ergologia não utiliza os conceitos de sujeito ou subjetividade, mas sim de "[...] 'corpo-si', árbitro no mais íntimo da atividade, [...] que não é um 'sujeito' delimitado, definido, mas uma entidade enigmática que resiste às tentativas de ser objetivado" (Schwartz: Duc \& Durrive, 2010, p. 196).

Conforme esses autores, o trabalhador é constantemente confrontado com variabilidades na realização de suas atividades, sendo-lhe impossível escapar de micro-escolhas rotineiras. Diante disso, o corpo-si é o árbitro e gestor dessas variabilidades que o impulsionam a escolher entre trabalhar "por si" ou "pelos outros", conduzindo, assim, seu trabalho. Reforça Schwartz, Duc e Durrive (2010), essa gestão é atravessada por economias do corpo, por sinalizações sensoriais e visuais, por um tipo de inteligência que passa pelo muscular, pelo neurofisiológico, mas que em seguida passa pela inconsciência do próprio corpo e pelo histórico. Consequentemente, a entidade que arbitra e decide não é inteiramente biológica, nem inteiramente consciente ou cultural, e é por isso que a ideia de corpo-si é posta pelos autores como preferível às noções de sujeito ou de subjetividade. Assim, tem-se a vantagem de não veicular "[...] certo número de possíveis malentendidos ou de evidências que criam obstáculos", uma vez que todo conceito carrega consigo uma história, apostas e valores (Schwartz; Duc \& Durrive, 2010, p. 197).

A abordagem ergológica consiste em um referencial teórico e metodológico profícuo para as pesquisas sobre o trabalho em saúde, a fertilidade da perspectiva teórico-metodológica da Ergologia para a análise do trabalho humano, e as características do trabalho em si, permite uma renovação de sentidos para o trabalho e para o trabalhador (Ribeiro \& Pires et. al, 2019; Santos \& Campomogara, 2014). A Ergologia não é uma disciplina no sentido de um novo domínio do saber, mas, antes de tudo, uma forma de pensamento, um modo de conceber a atividade humana (Schwartz, 2001) que se fundamenta em quatro pressupostos: i) pensar o geral e o específico, de acordo com a dialética entre o universal e o singular, que considera todas as formas de atividade; ii) articular diversas disciplinas, tais como a Linguística, a Antropologia, a Psicologia, a Economia e a Engenharia e interrogar seus saberes; iii) encontrar em todas as atividades as normas antecedentes e variabilidades, tanto as normas que são impostas quanto aquelas que se instalam, as renormalizações; iv) promover um regime de produção de saberes sobre o ser humano, porque o encontro entre os saberes científicos e práticos não pode ser previsto, pois sempre é uma descoberta (Brito, 2004).

Pelo modelo biomédico, o médico é o profissional central, e o trabalho em saúde organizado segundo os preceitos da divisão social e técnica do trabalho de tipo taylorista. A ergologia transgride o modelo biomédico, lança olhar sobre a necessidade de o trabalho em saúde possuir características predominantes do trabalho coletivo, envolvendo diversos trabalhadores e profissionais com saberes e conhecimentos específicos. O olhar da ergologia sobre o trabalho coletivo em 
saúde, procura alcançar um trabalho em que o resultado venha ter forte influência nas decisões e valores dos sujeitos que o realizam (Ribeiro \& Pires et. al, 2019; Santos \& Campomogara, 2014).

Durante o exercício das atividades laborais o modelo da ergologia permite ao profissional de saúde uma renovação de sentidos sobre o trabalho. Na prática diária o profissional de saúde possui embasamento científico para fazer escolhas, transgredir normas e modificar condutas. No tocante a renormalização e por vezes a ressingularização do processo de trabalho acontece através da singularidade de apenas o trabalhador projetar sua impressão de particularidades ao seu fazer, visando superar lacunas entre o trabalho prescrito e o real (Santos \& Campomogara, 2014).

A Ergologia considera que para toda a atividade de trabalho é depositado um saber individual, de cada ser humano, o que de certa forma, aproxima o prescrito do real. A ergologia considera que o trabalho prescrito é acometido por influências das características do meio, e outras "variabilidades permanentes"- como emoções, saberes, experiências, histórias etc., que o indivíduo possa sofrer, e ocasionar a persuasão da realização do trabalho. Seguindo, a ergologia trata o trabalho real como à atividade singular de um indivíduo, é a situação do ato do trabalho em si. Assim, a prescrição surge de uma ordenação desse trabalho (Ribeiro \& Pires et. al, 2019).

Uma premissa da Ergologia é de que ninguém pode falar do trabalho do outro sem antes ouvir aquele que realiza o trabalho. Uma possibilidade de aproximar-se da atividade é por meio da observação dos gestos (dos braços, das mãos, do rosto e de todo o corpo), dos movimentos, dos sentimentos, enfim dos saberes investidos no corpo si. São os saberes investidos, nem sempre conscientes, que orientam o corpo-si para responder às exigências do trabalho.

\subsection{Olhar da proteção radiológica sobre o trabalho em saúde}

O conceito amplo de "proteção radiológica" considera a prevenção dos possíveis danos provocados pelas radiações ionizantes, que dependem da dose de radiação absorvida, em consequência da carga de cada exposição e do acúmulo de cargas repetidas. Assim, é considerado como "radioproteção ou proteção radiológica" todo cuidado que reduz a exposição à radiação ionizante, tais como a indicação de exames radiológicos adequados e indispensáveis, a utilização da mínima dose necessária de radiação para realizar o exame com qualidade e restringindo a exposição as áreas de interesse e o uso de equipamentos de proteção individual para o técnico e para as pessoas que estão nas proximidades e, por algum motivo, não podem se afastar da fonte de radiação (Moores, 2017; Kase, 2004). A Comissão Internacional de Proteção Radiológica elege três princípios fundamentais que orientam os cuidados de radioproteção: justificativa (deve haver uma indicação médica precisa para realização do exame), otimização (cuidados relativos à realização do exame e controle dos profissionais mais expostos) e limitação da dose (a mínima necessária e suficiente para realizar o exame com qualidade (Kase, 2004).

As normas e a legislação de Radioproteção começaram a ser estabelecidas em 1928 e atualmente são bastante abrangentes, completas e universais, incluindo a brasileira (Moores, 2017; Brasil, 1998). Entretanto, raramente essas normas são observadas em sua totalidade, resultando em risco desnecessário aos trabalhadores e usuários dos serviços de saúde. Foi proposto pela Associação Internacional de Proteção Radiológica o conceito de "Cultura de Proteção Radiológica", que pode ser resumido como

a combinação de conhecimentos, valores, comportamentos e experiências de proteção radiológica em todos os seus aspectos para os pacientes, os trabalhadores da saúde, a população e ambiente em todas as situações de exposição à radiação, combinando as dimensões científicas e sociais (Ploussi \& Efstathopoulos, 2016).

Sobre a vivência nos serviços de atenção à saúde, observa-se o comportamento e atitudes dos profissionais em hospitais e clínicas radiológicas, compreende-se que as pessoas têm atitudes de proteção individual, mas não se percebe 
conhecimento e formação amparando-as, com os demais profissionais presentes no ambiente e com os usuários. Dessa forma, este estudo focaliza justamente as práticas e medidas de proteção de profissionais da saúde em contexto de Radioproteção.

Os trabalhadores que estão em contato com a radiação ionizante, como os profissionais das técnicas radiológicas (técnicos e tecnólogos em radiologia), são denominados trabalhadores ocupacionalmente expostos e os profissionais que participam de alguma forma da execução de exames de diagnóstico por imagem, auxiliando os profissionais das técnicas radiológicas, como enfermeiros, auxiliares e técnicos de enfermagem e médicos, serão denominados aqui trabalhadores paraocupacionalmente expostos, assim como a Norma Regulamentadora 32 (NR 32) denomina o trabalhador cujas atividades laborais não estão ligadas diretamente às radiações, mas que ocasionalmente podem receber doses superiores aos limites preconizados pela Norma Nuclear (NN) da Comissão Nacional de Energia Nuclear (CNEN), NN 3.01 (Brasil, 2005).

No Brasil, a necessidade de se estabelecer normas mais rigorosas nos serviços de radiodiagnóstico desencadeou-se pelo acidente ocorrido em Goiânia, em setembro de 1987, com um aparelho abandonado em um ferro velho que era, anteriormente, utilizado em tratamentos de radioterapia. Esse equipamento, que foi destruído por dois homens, possuía o elemento radioativo Césio-137 e causou o maior acidente radioativo do Brasil. O elemento em questão contaminou centenas de pessoas. Logo após a contaminação, quatro pessoas morreram. Esse acidente foi amplamente divulgado nacional e internacionalmente, dando início a vários ajustes e a implementação de novas práticas nos serviços de diagnóstico por imagem que envolvem radiação ionizante (Chemello, 2010).

As normas de Proteção Radiológica, no Brasil, datam do início de 1978, nas diretrizes da Segurança e Trabalho, determinadas pela Portaria ${ }^{\circ} 3.214$, de 8 de junho de 1978. Atualmente foi publicado a Resolução - RDC $n^{\circ} 330$, de 20 de dezembro de 2019, que revogou a Portaria SVS/MS nº 453 de $1^{\circ}$ de junho de 1998, que há duas décadas estabelecia as diretrizes básicas de Proteção Radiológica em radiodiagnóstico médico e odontológico, dispondo sobre o uso dos raios $\mathrm{X}$ diagnósticos em todo território nacional e dá outras providências. Sobre o ponto de vista de proteção radiológica, a RDC ${ }^{\circ}$ 330 emergiu para atualizar as normativas de clínicas, hospitais e outros serviços público ou privado, civil ou militar, que prestem serviços radiologia diagnóstica ou intervencionista, bem como os fabricantes ou comercializantes de equipamentos de radiologia, e instituições de ensino e pesquisa. Ainda a RDC $330 \mathrm{em}$ seu Artigos n⿳5, n $\mathrm{n}^{\circ} 17$ e n ${ }^{\circ} 42$, nos incisos III e I, exige dos serviços de radiologia diagnóstica e intervencionista um Memorial Descritivo (MD) aprovado pela vigilância sanitária, e que contenha um Programa de Proteção Radiológica (PPR), que contemple, no mínimo, medidas de prevenção, de controle e de vigilância e monitoramento, para garantir a segurança e a qualidade dos procedimentos radiológicos (Brasil, 2019).

O PPR deve ser incorporado ao Plano de Prevenção de Riscos Ambientais (PPRA) e incluir o risco físico "Radiação Ionizante", que deve ser reconhecido pela Comissão Interna de Prevenção de Acidentes (CIPA) e servir de base para a prevenção de acidentes no cotidiano de trabalho que envolve radiação ionizante ou materiais radioativos (Pro-Rad, 2013).

Além da contemporânea RDC 330, vale ressaltar a importância da NR 32, aprovada pela Portaria 483/2005, que estabelece um PPR com diretrizes básicas para a implementação de medidas de proteção a segurança e saúde de dos trabalhadores dos serviços de saúde e daqueles que exercem atividades de promoção e assistência à saúde em geral (Brasil, 2005).

\subsection{A proteção radiológica interpretada à luz da ergologia}

O incremento de estudos relacionados à prática assistencial e de pesquisa em Proteção Radiológica, abordando a interação dos trabalhadores e as situações reais de trabalho na perspectiva de renormatizações que motivam mudanças nas organizações podem contribuir com o deslocamento de ações de atenção e gestão da forma tradicional para modelos flexíveis, por sua característica de dinamismo e mutação. 
Na Proteção Radiológica, a utilização da perspectiva ergológica favorece a construção de um labor coletivo por meio de grupos de trabalho, evidenciando aspectos que permeiam o trabalho prescrito e o real relevante a ser considerado porque é nessa primeira instância das relações laborais que o trabalho da Proteção Radiológica se operacionaliza.

Essa interação, para permitir avanços positivos no ambiente de trabalho que favoreçam os trabalhadores e os usuários deve pautar-se na flexibilidade, no investimento, na escuta e diálogo e na inclusão dos sujeitos no processo decisório, o que, por conseguinte, amplia a autonomia.

Tais requisitos resgatam a essência do trabalhador e, concomitantemente, convoca o individual a construir e reconstruir os diferentes encontros entre os trabalhadores. Esse movimento do individual e coletivo, sempre variável perante uma norma antecedente, constitui mais uma evidência da possibilidade da utilização do referencial na proteção radiológica.

Compreendendo o trabalho enquanto construção individual e coletiva e valorizando o papel central do trabalhador nos processos de organização e gestão laboral, compreende-se que a ergologia pode contribuir na assistência e na construção de práticas organizacionais e formas de gestão mais flexíveis e humanizadas, como propõe, por exemplo, a Política Nacional de Humanização da Atenção e Gestão do Sistema Único de Saúde (Brasil, 2009), por meio da dramática do uso de si e da dialética que se promove ao direcionar o debate entre o trabalho prescrito e o trabalho real que perpassa a atividade de trabalho. O diferencial desta política reside também na clara preocupação com a capacitação e o desenvolvimento dos trabalhadores do setor saúde, de proporcionar condições adequadas para a execução das atividades laborativas e de criar condições para que os que cuidam possam ter suas necessidades satisfeitas (Brasil, 2009).

\section{Conclusão}

Buscou-se neste estudo discorrer e refletir acerca da Ergologia como referencial teórico e suas possibilidades para aplicabilidade na Proteção Radiológica nos serviços de saúde. Sabe-se que refletir sobre ergologia é desafiador, pois admite a ideia de que o trabalho é dinâmico, que têm forças divergentes e convergentes que dialogam entre si e se articulam, tanto no âmbito individual como coletivo e que o trabalhador é sujeito da própria história.

Pensar o trabalho de Proteção Radiológica sob a perspectiva da Ergologia é reconhecer que, pelas diversas dimensões que engloba, requer uma dialética das dramáticas do uso de si e do debate entre o trabalho prescrito e o trabalho real e, nesse sentido, ampliar o uso desse referencial na assistência e na pesquisa, maximizando a compreensão do processo de trabalho dos profissionais envolvidos.

Ressalta-se que escassos estudos foram encontrados na literatura, envolvendo a aplicabilidade da Ergologia na Proteção Radiológica. Assim, sugere-se a realização de outras investigações dessa natureza, em novos cenários, no intuito de se alcançar uma melhor compreensão das possibilidades para aplicabilidade da Ergologia na Proteção Radiológica nos serviços de saúde. Ainda, que se intensifique a execução da prática nessas áreas de atuação, pautadas na Ergologia, ampliando e maximizando a melhoria dos processos de trabalho, a assistência prestada aos pacientes bem como a qualidade de vida dos profissionais.

Dessa forma, espera-se que este estudo desperte o interesse acadêmico, a fim de ampliar os estudos nesta temática, assim como, a leitura deste material alcance os serviços de Proteção Radiológica e seus profissionais.

\section{Referências}

Athayde, M. \& Brito, J. (2011). Ergologia e clínica do trabalho. In: Bendassolli, P. F. \& Soboll, L. A. P. (Org.). Clínicas do trabalho: novas perspectivas para compreensão do trabalho na atualidade. Atlas, 2011. 258-281 p.

Batista, V. M. D.; Bernardo, M. O.; Morgado, F. \& Almeida, F. A. (2019). Proteção radiológica na perspectiva dos profissionais de saúde expostos à radiação. Rev Bras Enferm [Internet]. 72(1):12-9. https://doi.org/10.1590/0034-7167-2017-0545 
Borges, M. E. S. (2004). Trabalho e gestão de si: para além dos "recursos humanos". Cad. Psicol. Soc. Trab. 7: 41-9. <http://pepsic.bvsalud.org/scielo.php?script=sci_arttext\&pid=S1516-37172004000100005\&lng=en\&nrm=iso>.

Brand, C. I.; Fontana, R. T. \& Santos, A. V. dos. (2011). A saúde do trabalhador em radiologia: algumas considerações. Texto \& Contexto de Enf., 20 (1): 68 75. https://doi.org/10.1590/S0104-07072011000100008

Brasil. (1998). Secretaria de Vigilância Sanitária do Ministério da Saúde. Portaria $n^{\circ} 453$ de $1^{\circ}$ de junho de 1998. Diretrizes de proteção radiológica em radiodiagnóstico médico e odontológico. Brasília: Diário Oficial da União.

Brasil. (2009). Ministério da Saúde. Secretaria de Atenção à Saúde. Política Nacional de Humanização da Atenção e Gestão do SUS. Gestão participativa e cogestão. Brasília [citado 2011 fev. 2]. Disponível em: http://bvsms.saude.gov.br/bvs/publicacoes/gestao_participativa_cogestao.pdf.

Brasil. (2019). Ministério da Saúde. Resolução - RDC $n^{\circ}$ 330, de 20 de dezembro de 2019. Resolução que estabelece os requisitos sanitários para a organização e o funcionamento de serviços de radiologia diagnóstica ou intervencionista e regulamenta o controle das exposições médicas, ocupacionais e do público decorrentes do uso de tecnologias radiológicas diagnósticas ou intervencionistas. Ministério da Saúde/Agência Nacional de Vigilância Sanitária/Diretoria Colegiada. Diário Oficial da União. Publicado em: 26/12/2019 | Edição: 249 | Seção: 1 | Página: 92

Brito, J. (2004). Saúde do trabalhador: reflexões a partir da abordagem ergológica. In: Figueiredo, M.; Athayde, M., Brito, J. \& Alvarez, D. organizadores. Labirintos do trabalho: interrogações e olhares sobre o trabalho vivo.: DP\&A. p. 91-114.

Campos, L. F.; Melo, M. R. A. C. \& Telles Filho, P. C. P. (2014). Ergologia como referencial teórico: possibilidades para assistência e pesquisa em enfermagem. R. Enferm. Cent. O. Min. maio/ago; 4(2):1222-1228. https://doi.org/10.19175/recom.v0i0.613

CNEN. (2006). Comissão Nacional de Energia Nuclear, CNEN-NN-3.01, Diretrizes básicas de proteção radiológica. Portaria CNEN/PR 007 de 17/01/2006 (Alterações). Publicada em Diário Oficial da União em 18/01/2006.

Durrive, 1. \& Jacques, A. M. O formador ergológico ou "Ergoformador": uma introdução à ergoformação In: In: Schwartz, Y.; Durrive, L. (Org.). Trabalho \& Ergologia: conversas sobre a atividade humana. (2a ed.), Niterói: EdUFF, 2010. 295-307.

Fernandes, G. S. et. al. (2005). Avaliação dos riscos ocupacionais de trabalhadores de serviço de radiologia. Radiol. Bras. 38 (4). https://doi.org/10.1590/S0100-39842005000400009

Fernandes, C. C. (2019). Proteção radiológica de radiodiagnósticos médicos - um estudo de caso em ambiente hospitalar no Rio de Janeiro. Braz. J. of Bus. Curitiba, 1 (2): 546-563

Figueiredo, F. M. de \& Gama, Z. A. S. (2012). Melhoria da proteção radiológica mediante um ciclo de avaliação interna da qualidade. Radiol. Bras.; 40 (2): 87-92. https://doi.org/10.1590/S0100-39842012000200005

Flôr, R. C.; Melo, J. A. C. de; Gelbcke, F. L.; Ramos, F. R. S. \& Amadigi, F. R. (2017). Investigação da práxis em enfermagem radiológica: aplicação da metodologia da psicodinâmica do trabalho. Texto \& Contexto Enf.; 26 (3), e0930017. https://doi.org/10.1590/0104-07072017000930017

Flôr, R. C.; Melo, J. A. C. de \& Gelbcke, F. L. (2013). Proteção radiológica e a atitude de trabalhadores de enfermagem em serviço de hemodinâmica. Texto \& Contexto Enf:; 22 (2): 416-22. https://doi.org/10.1590/S0104-07072013000200018

Hennington, A. F.; Cunha, D. M. \& Fischer, M. C. B. (2011). Trabalho, educação, saúde e outros possíveis: diálogos na perspectiva ergológica. Trab. Educ. Saúde, Rio de Janeiro, 9 (supl. 1): 5-18. https://doi.org/10.1590/S1981-77462011000400001

Hennington, E. A. (2008). Gestão de processos de trabalho e humanização em saúde: reflexões a partir da ergologia. Rev. Saúde Pública. 42(3): 555-61. https://doi.org/10.1590/S0034-89102008005000022

Holz, E. B. \& Bianco, M. F. (2014). Ergologia: uma abordagem possível para os estudos organizacionais sobre trabalho. Cad. EBAPE.BR, 12: 494-512, Edição Especial, artigo 6.

Huhn, A.; Vargas, M. A. O; Melo, J. A. C.; Gelbcke, F. L.; Ferreira, M. L.; Lança, L. (2017). Implementação do programa de proteção radiológica: Olhar da equipe de saúde atuante em um serviço de Radiologia. Texto Contexto Enferm. 26(1): e5370015https://doi.org/10.1590/0104-07072017005370015

ICRP. (2002). International Commission on Radiological Protection. Recommendations of the International Commission on Radiological Protection, ICRP Publication 60, Pergamon Press, New York.

Kase, K. R. (2004). Radiation protection principles of NCRP. Health Phys. 87(3):251-7. 10.1097/00004032-200409000-00005.

Lima, E. L. N. \& Bianco, M. F. (2009). Análise de situações de trabalho: gestão e os usos de si dos trabalhadores de uma empresa do ramo petrolífero. Rio de Janeiro: Cadernos EBAPE. 7(4): 632-58.

Mendes, K. D. S.; Silveira, R. C. C. P. \& Galvão, C. M. (2008). Revisão integrativa: método de pesquisa para a incorporação de evidência na saúde e na enfermagem. Texto \& contexto enferm. 17(4):758-64. 10.1590/S0104-07072008000400018

Moores, B. M. (2017). A review of the fundamental Principles of radiation protection when applied to the patient in diagnostoc radiology. Radiat Prot Dosimetry. 175(1):1-9. 10.1093/rpd/ncw259.

Neves, T. P. (2008). As contribuições da ergologia para a compreensão da biossegurança como processo educativo: perspectivas para a saúde ambiental e do trabalhador. O Mundo da Saúde 32(3):367-375.

Pereira, L. (2004). Caracterização e implantação de feixes de radiação para estudo de dispositivos de proteção individual com equivalência em chumbo utilizados em práticas de radiodiagnóstico. Dissertação (Mestrado) - Instituto de Radioproteção e Dosimetria. 
Research, Society and Development, v. 10, n. 7, e56610716886, 2021

(CC BY 4.0) | ISSN 2525-3409 | DOI: http://dx.doi.org/10.33448/rsd-v10i7.16886

Pereira A. S. et al. (2018). Metodologia da pesquisa científica. [free e-book]. Santa Maria/RS. Ed. UAB/NTE/UFSM. 5.3) Koche, J. C. (2011). Fundamentos de metodologia científica. Petrópolis: Vozes. Disponível em: http://www.brunovivas.com/wp-content/uploads/sites/10/2018/07/K\%C3\%B6che-Jos\%C3\%A9Carlos0D0AFundamentos-de-metodologia-cient\%C3\%ADfica-_teoria-da0D0Aci\%C3\%AAncia-e-inicia\%C3\%A7\%C3\%A3o-\%C3\%A0- pesquisa. pdfhttps://repositorio.ufsm.br/bitstream/handle/1/15824/Lic_Computacao_Metodologia-Pesquisa-Cientifica.pdf?sequence=15.4)

Ploussi, A. \& Efstathopoulos, E. P. (2016). Importance of establishing radiation protection culture in Radiology Department. World J Radiol. 8 (2):142-7. doi: 10.4329/wjr. v8.i2.142.

Pro-Rad. (2013). Proteção Radiológica. Consultores em radioproteção $\quad$ S/S $\quad$ Ltda $\quad$ [Internet]. 2013 http://www.prorad.com. br/index.php?data=memorial_descritivo.php

Pompeo, D. A.; Rossi, L. A. \& Galvão, C. M. (2009). Revisão integrativa: etapa inicial do processo de validação de diagnóstico de enfermagem. Acta paul enferm. 22(4):434. https://doi.org/10.1590/S0103-21002009000400014

Querido, F. M. \& Poveda, V. B. (2015). Exposição da equipe de enfermagem à radiação em centro cirúrgico: um estudo descritivo. Rev. SOBECC, 20 (1): 2-8.

Ribeiro, G. \& Pires, D. E. P. et al. Construções tórico-metodológicas da ergologia para a pesquisa sobre o trabalho na área de enfermagem. Texto contexto enferm., $\quad$ e20170163, <http://www.scielo.br/scielo.php?script=sci_arttext\&pid=S0104-07072019000100611\&lng=en\&nrm=iso>.. https://doi.org/10.1590/1980-265x-tce-2017-0163.

Santos, T. M. dos \& Camponogara, S. (2014). Um olhar sobre o trabalho de enfermagem e a ergologia. Trabalho, Educação e Saúde. 12 (1): 149-163. https://doi.org/10.1590/S1981-77462014000100009

Schwartz, Y. (2001). Trabalho e educação. Presença Pedagógica. 7(38):5-17.

Schwartz, Y. (2006). Entrevista. Rev. Trabalho, educação e saúde. 4(2): 457-66.

Schwartz, Y. \& Durrive, L. (Org.). (2010). Trabalho \& Ergologia: conversas sobre a atividade humana. (2a ed.), EdUFF, p. 295-307.

Silveira, C. S. \& Zago, M. M. F. (2006). Pesquisa brasileira em enfermagem oncológica: uma revisão integrativa. Rev latinoam enferm. 14(4):614-9. $10.1590 / \mathrm{S} 0104-11692006000400021$ 